\title{
Exponential Stability of Impulsive Stochastic Functional Differential Systems with Delayed Impulses
}

\author{
Fengqi Yao, ${ }^{1}$ Feiqi Deng, ${ }^{2}$ and Pei Cheng ${ }^{3}$ \\ ${ }^{1}$ School of Electrical Engineering \& Information, Anhui University of Technology, Ma'anshan, Anhui 243000, China \\ ${ }^{2}$ The Institute of System Engineering, South China University of Technology, Guangzhou, Guangdong 510640, China \\ ${ }^{3}$ School of Mathematical Science, Anhui University, Hefei, Anhui 230039, China \\ Correspondence should be addressed to Fengqi Yao; fqyao85@ahut.edu.cn
}

Received 6 December 2012; Revised 24 February 2013; Accepted 11 March 2013

Academic Editor: Cristina Pignotti

Copyright (C) 2013 Fengqi Yao et al. This is an open access article distributed under the Creative Commons Attribution License, which permits unrestricted use, distribution, and reproduction in any medium, provided the original work is properly cited.

\begin{abstract}
A class of generalized impulsive stochastic functional differential systems with delayed impulses is considered. By employing piecewise continuous Lyapunov functions and the Razumikhin techniques, several criteria on the exponential stability and uniform stability in terms of two measures for the mentioned systems are obtained, which show that unstable stochastic functional differential systems may be stabilized by appropriate delayed impulses. Based on the stability results, delayed impulsive controllers which mean square exponentially stabilize linear stochastic delay systems are proposed. Finally, numerical examples are given to verify the effectiveness and advantages of our results.
\end{abstract}

\section{Introduction}

In recent years, the theory of impulsive functional differential systems (IFDSs) has attracted an increasing interest due to the wide existence of impulse effects and time delays in realworld systems. An area of particular interest has been the impulsive control of delay systems, with consequent emphasis on stability analysis of IFDSs; see [1-8] and reference therein. However, in the current literature on IFDSs, most authors have assumed that the impulses are only related to the present states. But in most cases, it is more realistic that the impulses depend not only on the present but, also the past states, and such impulses are called delayed impulses. Recently, several studies have attempted to investigate IFDSs with delayed impulses (IFDSs-DI); see [9-12]. By employing Lyapunov functions coupled with Razumikhin techniques, [9] investigated the asymptotic stability and practical stability for a class of generalized IFDSs-DI, while [10, 11] further established several criteria for the exponential stability of the systems. In [12], sufficient conditions for the stability of impulsive differential systems with linear delayed impulses were derived and then applied to impulsively synchronize two coupled chaotic systems by using delayed impulses.
In addition to impulse effects and time delays, as is well known, environment noise exists inevitably in real systems and may greatly affect the performance of systems. Recently, $[13,14]$ took environment noise into account and generalized delayed impulses to stochastic systems. In particular, applying the Lyapunov-Razumikhin techniques, [13] investigated both moment and almost sure exponential stability of impulsive stochastic functional differential systems with delayed impulses (ISFDSs-DI). In [14], the authors started the study of robust stability and state-feedback stabilization of uncertain impulsive stochastic delayed differential systems with linear delayed impulses.

On the other hand, impulsive control has become an active research area and found successful applications in a wide variety of areas, such as control and synchronization of chaotic systems [15-17], ecosystems management [18], secure communication [15], and orbital transfer of satellite [19, 20]. In the past few years, the impulsive control theory has been generalized from deterministic systems to stochastic systems; see $[13,21,22]$. But in many cases, some well-designed impulsive control schemes cannot work as expected. One reason is that the schemes designed are usually free of delays, but time delays do exist due to time spent in computation and transfer. 
As is well known, the presence of time delays of controllers may be the cause of serious deterioration of performance or even instability of the resulting controlled system if it is not considered in controller design [23]. One way to overcome this problem is to use delayed impulsive control laws.

Motivated by the above discussion, the present paper will employ the Razumikhin techniques to investigate the problems of stability analysis and impulsive stabilization of ISFDSs-DI. Obviously, it is more difficult to motivate ISFDSsDI than the common ISFDSs with nondelayed impulses (ISFDSs-nDI), and the key difficulties and challenges lie in finding proper way to deal with the delayed impulses. Different from [13], we will exploit the notion of supremum to deal with the delayed impulses, which will relaxe the restriction imposed on impulses. The proof of the paper is enlightened by the idea from $[6,10]$. Our results extend and generalize some results existing in the literature and show that appropriate delayed impulsive perturbations may make unstable stochastic functional differential systems stable. The rest of the paper is organized as follows. In Section 2, we introduce some notations and definitions. In Section 3, several criteria on the exponential stability and uniform stability in terms of two measures for ISFDSs-DI are established. Then, Section 4 is devoted to the discussion of two special types of ISFDSsDI, which is followed by the stabilization of linear stochastic delay systems by using delay impulses in Section 5. Finally, illustrative examples and concluding remarks are given in Section 6 and Section 7, respectively.

\section{Preliminaries}

Throughout this paper, unless otherwise specified, we will employ the following notations. Let $\left(\Omega, \mathscr{F},\left\{\mathscr{F}_{t}\right\}_{t \geqslant 0}, \mathbb{P}\right)$ be a complete probability space with a filtration $\left\{\mathscr{F}_{t}\right\}_{t \geqslant 0}$ satisfying the usual conditions (i.e., it is right continuous, and $\mathscr{F}_{0}$ contains all $\mathbb{P}$-null sets), and let $\mathbb{E}[\cdot]$ be the expectation operator with respect to the probability measure. Let $B(t)=$ $\left(B_{1}(t), \ldots, B_{m}(t)\right)^{\mathrm{T}}$ be an $m$-dimensional Brownian motion defined on the probability space. $\mathbb{R}=(-\infty,+\infty), \mathbb{R}_{+}=$ $[0,+\infty), \mathbb{N}=\{1,2,3, \ldots\}$, and $\mathbb{R}^{n}$ denotes the $n$-dimensional real space equipped with the Euclidean norm $|\cdot|$. Moreover, $\Gamma=\left\{h:\left[t_{0}-\tau, \infty\right) \times \mathbb{R}^{n} \rightarrow \mathbb{R}_{+} \mid h(t, x)\right.$ is continuous with $\left.\inf _{(t, x)} h(t, x)=0\right\}$.

Let $\tau \geqslant 0$, and $P C\left([-\tau, 0] ; \mathbb{R}^{n}\right)=\left\{\varphi:[-\tau, 0] \rightarrow \mathbb{R}^{n} \mid \varphi(t)\right.$ is continuous for all but at most a finite number of points $\bar{t}$ at which $\varphi\left(\bar{t}^{+}\right), \varphi\left(\bar{t}^{-}\right)$exist, and $\left.\varphi\left(\bar{t}^{+}\right)=\varphi(\bar{t})\right\}$ with the norm $\|\varphi\|=\sup \{|\varphi(\theta)|:-\tau \leqslant \theta \leqslant 0\}$, where $\varphi\left(\bar{t}^{+}\right)$and $\varphi(\bar{t})$ denote the right-hand and left-hand limits of $\varphi(t)$ at $\bar{t}$, respectively. Let $P C_{\mathscr{F}_{t}}^{b}\left([-\tau, 0] ; \mathbb{R}^{n}\right)$ be the family of all $\mathscr{F}_{t^{-}}$ measurable and bounded $P C\left([-\tau, 0] ; \mathbb{R}^{n}\right)$-valued random variables $\xi=\{\xi(\theta):-\tau \leqslant \theta \leqslant 0\}$.

If $A$ is a vector or matrix, its transpose is denoted by $A^{\mathrm{T}}$. If $P$ is a square matrix, then $P>0(P<0)$ means that $P$ is a symmetric positive (negative) definite matrix, while $P \geqslant$ $0(P \leqslant 0)$ is a symmetric positive (negative) semidefinite matrix. $\lambda_{\min }(\cdot)$ and $\lambda_{\max }(\cdot)$ represent the minimum and maximum eigenvalues of the corresponding matrix, respectively, and $I$ stands for the identity matrix. Unless explicitly stated, all matrices are assumed to have real entries and compatible dimensions.

Let us consider the following ISFDSs-DI as follows:

$$
\begin{gathered}
\mathrm{d} x(t)=f\left(t, x_{t}\right) \mathrm{d} t+\sigma\left(t, x_{t}\right) \mathrm{d} B(t), \quad t \geqslant t_{0}, \quad t \neq t_{k}, \\
x\left(t_{k}\right)=I_{k}\left(t_{k}, x_{t_{k}^{-}}\right), \quad k \in \mathbb{N},
\end{gathered}
$$

with initial value $x_{t_{0}}=\xi=\{\xi(\theta):-\tau \leqslant \theta \leqslant 0\} \in P C_{\mathscr{F}_{t_{0}}}^{b}([-\tau$, $\left.0] ; \mathbb{R}^{n}\right)$, where $x \in \mathbb{R}^{n} ; x_{t}=\{x(t+\theta):-\tau \leqslant \theta \leqslant 0\}$ is regarded as a $P C\left([-\tau, 0] ; \mathbb{R}^{n}\right)$-valued random variables. Moreover, $f$ : $\mathbb{R}_{+} \times P C\left([-\tau, 0] ; \mathbb{R}^{n}\right) \rightarrow \mathbb{R}^{n}$ and $\sigma: \mathbb{R}_{+} \times P C([-\tau, 0] ;$ $\left.\mathbb{R}^{n}\right) \rightarrow \mathbb{R}^{n \times m}$ are Borel measurable; $I_{k}\left(t_{k}, x_{t_{k}^{-}}\right): \mathbb{R}_{+} \times P C([-\tau$, $\left.0] ; \mathbb{R}^{n}\right) \rightarrow \mathbb{R}^{n}$ represents the impulsive perturbation of $x$ at time $t_{k}$. The fixed moments of time $t_{k}$ satisfy $0 \leqslant t_{0}<t_{1}<$ $\cdots<t_{k}<\cdots, \lim _{k \rightarrow \infty} t_{k}=\infty$.

As a standing hypothesis, we assume that for any $\xi \in$ $P C_{\mathscr{F}_{t_{0}}}^{b}\left([-\tau, 0] ; \mathbb{R}^{n}\right)$, there exists a unique solution to system (1) denoted by $x\left(t ; t_{0}, \xi\right)$ [24], which is continuous except at $t=t_{k}$, at which it is right continuous and left limitable. For the purpose of stability analysis, we further assume that $f(t, 0)=\sigma(t, 0)=I_{k}(t, 0) \equiv 0$ for all $t \geqslant t_{0}, k \in \mathbb{N}$, then system (1) admits a trivial solution $x(t) \equiv 0$.

Definition 1 (see [25]). A function $V(t, x):\left[t_{0}-\tau, \infty\right) \times$ $\mathbb{R}^{n} \rightarrow \mathbb{R}_{+}$is said to belong to the class $v_{0}$, if $V$ is continuous on each of the sets $\left[t_{k-1}, t_{k}\right) \times \mathbb{R}^{n}, \lim _{(t, y) \rightarrow\left(t_{k}^{-}, x\right)} V(t, y)=$ $V\left(t_{k}^{-}, x\right)$ exists, and $V_{t}, V_{x}$, and $V_{x x}$ are continuous for $(t, x) \in$ $\left(t_{k-1}, t_{k}\right) \times \mathbb{R}^{n}, k \in \mathbb{N}$, where

$$
\begin{gathered}
V_{t}(t, x)=\frac{\partial V(t, x)}{\partial t}, \\
V_{x}(t, x)=\left[\frac{\partial V(t, x)}{\partial x_{1}}, \ldots, \frac{\partial V(t, x)}{\partial x_{n}}\right], \\
V_{x x}(t, x)=\left[\frac{\partial^{2} V(t, x)}{\partial x_{i} \partial x_{j}}\right]_{n \times n} .
\end{gathered}
$$

For each $V \in v_{0}$ and $(t, \phi) \in\left[t_{k-1}, t_{k}\right) \times P C_{\mathscr{F}_{t}}^{b}([-\tau, 0]$; $\left.\mathbb{R}^{n}\right),(k \in \mathbb{N})$, one defines the Kolmogorov operator $\mathscr{L} V$ associated with system (1) by

$$
\begin{aligned}
\mathscr{L} V(t, \phi)= & V_{t}(t, \phi(0))+V_{x}(t, \phi(0)) f(t, \phi) \\
& +\frac{1}{2} \operatorname{trace}\left[\sigma^{\mathrm{T}}(t, \phi) V_{x x}(t, \phi(0)) \sigma(t, \phi)\right] .
\end{aligned}
$$

Definition 2 (see [4]). Let $h^{0} \in \Gamma, \varphi \in P C\left([-\tau, 0] ; \mathbb{R}^{n}\right)$. One defines

$$
h_{0}(t, \varphi)=\sup _{-\tau \leqslant s \leqslant 0} h^{0}(t+s, \varphi(s)), \quad t \geqslant t_{0} .
$$

Definition 3. Let $h^{0}, h \in \Gamma$. Then, system (1) is said to be

(S1) $\left(h_{0}, h\right)$-uniformly stable, if for any given $\varepsilon>0$ and $t_{0} \in \mathbb{R}_{+}$, there exists a $\delta=\delta(\varepsilon)>0$ such that

$\mathbb{E} h_{0}\left(t_{0}, \xi\right)<\delta \quad$ implies $\mathbb{E} h\left(t, x\left(t ; t_{0}, \xi\right)\right)<\varepsilon, \quad t \geqslant t_{0}$, 
(S2) $\left(h_{0}, h\right)$-globally exponentially stable, if there exist constants $\alpha>0$ and $C \geqslant 1$ such that, for all $\xi \in$ $P C_{\mathscr{F}_{t_{0}}}^{b}\left([-\tau, 0] ; \mathbb{R}^{n}\right)$ and $t_{0} \in \mathbb{R}_{+}$,

$$
\mathbb{E} h\left(t, x\left(t ; t_{0}, \xi\right)\right) \leqslant C \mathbb{E} h_{0}\left(t_{0}, \xi\right) \mathrm{e}^{-\alpha\left(t-t_{0}\right)}, \quad t \geqslant t_{0} .
$$

Remark 4. The $\left(h_{0}, h\right)$-stability notions are considered here in the spirit of the work by Lakshmikantham and Liu [26] to unify different stability concepts found in the literature such as the stability of the trivial solution, the partial stability, the conditional stability, and the stability of invariant sets, which would otherwise be treated separately. For example, it is easy to see that (S2) in Definition 3 gives

(1) the $p$ th-moment exponential stability of the trivial solution $x(t) \equiv 0$, if $h(t, x)=h^{0}(t, x)=|x|^{p}$. When $p=2$, it is usually called mean square exponential stability;

(2) the pth-moment exponentially partial stability of the trivial solution, if $h(t, x)=\left|\left(x_{1}, \ldots, x_{s}\right)\right|^{p}, 1 \leqslant s \leqslant n$ and $h^{0}(t, x)=|x|^{p}$;

(3) the global exponential stability of the prescribed motion $y(t)$, if $h(t, x)=h^{0}(t, x)=|x-y|$;

(4) the global exponential stability of the invariant set $A \in$ $\mathbb{R}^{n}$, if $h(t, x)=h^{0}(t, x)=d(x, A)$, where $d(x, A)$ is the distance of $x$ from the set $A$;

(5) the global exponential orbital stability of a periodic solution, if $h(t, x)=h^{0}(t, x)=d(x, C)$, where $C$ is the closed orbit in the phase space.

\section{Stability Results}

In this section, we will develop Lyapunov-Razumikhin methods and establish some criteria which provide sufficient conditions for the $\left(h_{0}, h\right)$-exponential stability and $\left(h_{0}, h\right)$ uniform stability of ISFDSs-DI. Our results illustrate that the impulses play a positive role in making the continuous flow stable.

Theorem 5. Assume that there exist functions $V \in v_{0}, h^{0}, h \in$ $\Gamma$ and constants $c_{1}>0, c_{2}>0, \gamma>q>1$ such that

(i) $c_{1} h(t, x) \leqslant V(t, x) \leqslant c_{2} h^{0}(t, x)$ for any $(t, x) \in\left[t_{0}-\right.$ $\tau, \infty) \times \mathbb{R}^{n} ;$

(ii) $\mathbb{E} \mathscr{L} V(t, \phi) \leqslant b(t) c(\mathbb{E} V(t, \phi(0)))$ for all $t \in\left[t_{k-1}, t_{k}\right)$, $k \in \mathbb{N}$ and those $\phi \in P C_{\mathscr{F}_{t}}\left([-\tau, 0] ; \mathbb{R}^{n}\right)$ satisfying

$\mathbb{E} V(t+\theta, \phi(\theta)) \leqslant \gamma \mathbb{E} V(t, \phi(0)) \quad$ on $-\tau \leqslant \theta \leqslant 0$,

where $b, c: \mathbb{R}_{+} \rightarrow \mathbb{R}_{+}$are continuous;

(iii) $\mathbb{E} V\left(t_{k}, I_{k}\left(t_{k}, \phi\right)\right) \leqslant(1 / \gamma)\left(1+\beta_{k}\right) \sup _{s \in[-\tau, 0]} \mathbb{E} V\left(t_{k}^{-}+s\right.$, $\phi(s))$, where $\beta_{k} \geqslant 0$ and $\sum_{k=1}^{\infty} \beta_{k}<\infty$;

(iv) $\ln q>M_{1} M_{2}$, where $M_{1}=\sup _{t \geqslant 0} \int_{t}^{t+\mu} b(s) d s, M_{2}=$ $\sup _{s>0}\{c(s) / s\}$, and $\mu=\sup _{k \in \mathbb{N}}\left\{t_{k}-t_{k-1}\right\}$.

Then, system (1) is $\left(h_{0}, h\right)$-exponentially stable for any time delay $\tau \in(0, \infty)$, and the convergence rate is not greater than $\min \left\{\ln (\gamma / q) / \tau,\left(\ln q-M_{1} M_{2}\right) / \mu\right\}$.
Proof. Fix any initial data $\xi \in P C_{\mathscr{F}_{t_{0}}}^{b}\left([-\tau, 0) ; \mathbb{R}^{n}\right)$, and write $x\left(t ; t_{0}, \xi\right)=x(t)$ simply. Let $\beta>1$ be an arbitrary constant, and define $\alpha=\min \left\{\ln (\gamma / q) / \tau,\left(\ln q-M_{1} M_{2}\right) / \beta \mu\right\}$. We claim that

$$
\mathbb{E} V(t, x(t)) \leqslant q c_{2} \mathbb{E} h_{0}\left(t_{0}, \xi\right) H(t) \mathrm{e}^{-\alpha\left(t-t_{0}\right)}, \quad t \geqslant t_{0},
$$

where

$$
H(t)= \begin{cases}1, & t_{0}-\tau \leqslant t<t_{1}, \\ \prod_{t_{0}<t_{k} \leqslant t}\left(1+\beta_{k}\right), & t \geqslant t_{1} .\end{cases}
$$

Obviously, $H(t)$ is nondecreasing and

$$
\begin{gathered}
H(t)=H\left(t_{m-1}\right) \quad \text { for } t \in\left[t_{m-1}, t_{m}\right), m \in \mathbb{N}, \\
H(t)=1 \text { for } t \in\left[t_{0}, t_{1}\right) .
\end{gathered}
$$

For convenience, we write $\mathbb{E} V(t, x(t))=v(t)$ and $q c_{2} \mathbb{E} h_{0}\left(t_{0}\right.$, $\xi) H(t) \mathrm{e}^{-\alpha\left(t-t_{0}\right)}=w(t)$. Then, (8) can be written as

$$
v(t) \leqslant w(t), \quad t \geqslant t_{0} .
$$

We first prove that

$$
v(t) \leqslant w(t), \quad t \in\left[t_{0}, t_{1}\right) .
$$

If (12) is not true, there would be some $t \in\left[t_{0}, t_{1}\right)$ such that $v(t)>w(t)$. Let $t^{*}=\inf \left\{t \in\left[t_{0}, t_{1}\right): v(t)>w(t)\right\}$. Due to the continuity of $v(t)$ on $\left[t_{0}, t_{1}\right)$ and

$$
\begin{array}{r}
v\left(t_{0}+\theta\right) \leqslant c_{2} \mathbb{E} h_{0}\left(t_{0}, \xi\right) \leqslant \frac{1}{q} w\left(t_{0}+\theta\right)<w\left(t_{0}+\theta\right), \\
\theta \in[-\tau, 0],
\end{array}
$$

we get

$$
\begin{array}{r}
t^{*}>t_{0}, \quad v\left(t^{*}\right)=w\left(t^{*}\right), \quad v(t) \leqslant w(t) \\
\text { for } t \in\left[t_{0}, t^{*}\right) .
\end{array}
$$

Noticing $v\left(t^{*}\right)=w\left(t^{*}\right)>(1 / q) w\left(t^{*}\right), v\left(t_{0}\right) \leqslant(1 / q) w\left(t_{0}\right)$, we further define $t^{* *}=\sup \left\{t \in\left[t_{0}, t^{*}\right): v(t) \leqslant(1 / q) w(t)\right\}$, then

$$
\begin{array}{r}
t^{*}>t^{* *}, \quad v\left(t^{* *}\right)=\frac{1}{q} w\left(t^{* *}\right), \quad v(t)>\frac{1}{q} w(t) \\
\text { for } t \in\left(t^{* *}, t^{*}\right] .
\end{array}
$$

Hence,

$$
\frac{1}{q} w(t) \leqslant v(t) \leqslant w(t), \quad t \in\left[t^{* *}, t^{*}\right] .
$$

Consequently, since $t+\theta \in\left[t_{0}-\tau, t^{*}\right]$ for any $t \in\left[t^{* *}, t^{*}\right]$ and $\theta \in[-\tau, 0]$, it follows from (13), (14), and (16) that

$$
\begin{array}{r}
v(t+\theta) \leqslant w(t+\theta) \leqslant \mathrm{e}^{\alpha \tau} w(t) \leqslant q \mathrm{e}^{\alpha \tau} v(t) \leqslant \gamma v(t), \\
t \in\left[t^{* *}, t^{*}\right], \quad \theta \in[-\tau, 0],
\end{array}
$$


which, by condition (ii), implies that

$$
\mathbb{E} \mathscr{L} V\left(t, x_{t}\right) \leqslant b(t) c(v(t)) \leqslant M_{2} b(t) v(t), \quad t \in\left[t^{* *}, t^{*}\right] .
$$

By the Itô formula and applying the Gronwall inequality, we can derive that

$$
v\left(t^{*}\right) \leqslant v\left(t^{* *}\right) \mathrm{e}^{\int_{t^{* *}}^{t^{*}} M_{2} b(s) \mathrm{d} s} \leqslant v\left(t^{* *}\right) \mathrm{e}^{M_{1} M_{2}} .
$$

That is,

$$
q c_{2} \mathbb{E} h_{0}\left(t_{0}, \xi\right) \mathrm{e}^{-\alpha\left(t^{*}-t_{0}\right)} \leqslant c_{2} \mathbb{E} h_{0}\left(t_{0}, \xi\right) \mathrm{e}^{-\alpha\left(t^{* *}-t_{0}\right)+M_{1} M_{2}},
$$

which leads to

$$
\ln q \leqslant \alpha \mu+M_{1} M_{2}
$$

This contradicts the definition of $\alpha$. Hence, (12) holds.

Now, we assume that

$$
\begin{aligned}
& v(t) \leqslant w(t), \quad t \in\left[t_{k-1}, t_{k}\right), \\
& k=1,2, \ldots, m(m \in \mathbb{N}, m \geqslant 1) .
\end{aligned}
$$

Then, the following inequality holds by condition, (iii), (13), and (22) as follows:

$$
\begin{aligned}
v\left(t_{m}\right) & \leqslant \frac{1}{\gamma}\left(1+\beta_{m}\right) \sup _{s \in[-\tau, 0]} v\left(t_{m}^{-}+s\right) \\
& \leqslant \frac{1}{\gamma}\left(1+\beta_{m}\right) \sup _{s \in[-\tau, 0]} w\left(t_{m}^{-}+s\right) \\
& \leqslant \frac{1}{\gamma}\left(1+\beta_{m}\right) q c_{2} \mathbb{E} h_{0}\left(t_{0}, \xi\right) \mathrm{e}^{\alpha \tau} H\left(t_{m}^{-}\right) \mathrm{e}^{-\alpha\left(t_{m}-t_{0}\right)} \\
& \leqslant c_{2} \mathbb{E} h_{0}\left(t_{0}, \xi\right) H\left(t_{m}\right) \mathrm{e}^{-\alpha\left(t_{m}-t_{0}\right)}=\frac{1}{q} w\left(t_{m}\right)<w\left(t_{m}\right) .
\end{aligned}
$$

Next, we proceed to prove that

$$
v(t) \leqslant w(t), \quad t \in\left[t_{m}, t_{m+1}\right) .
$$

On the contrary, we suppose that (24) is not true. Then, there exist some $t \in\left[t_{m}, t_{m+1}\right)$ such that $v(t)>w(t)$. Setting $\bar{t}=$ $\inf \left\{t \in\left[t_{m}, t_{m+1}\right): v(t)>w(t)\right\}$, it follows from (23) and the continuity of $v(t)$ on $\left[t_{m}, t_{m+1}\right)$ that

$$
\bar{t}>t_{m}, \quad v(\bar{t})=w(\bar{t}), \quad v(t) \leqslant w(t) \quad \text { for } t \in\left[t_{m}, \bar{t}\right) .
$$

In view of $v(\bar{t})=w(\bar{t})>(1 / q) w(t)$ and $v\left(t_{m}\right) \leqslant(1 / q) w\left(t_{m}\right)$, we further define $\underline{t}=\sup \left\{t \in\left[t_{m}, \bar{t}\right): v(t) \leqslant(1 / q) w(t)\right\}$, then

$$
\underline{t}<\bar{t}, \quad v(\underline{t})=\frac{1}{q} w(\underline{t}), \quad v(t)>\frac{1}{q} w(t) \quad \text { for } t \in(\underline{t}, \bar{t}] .
$$

Thus,

$$
\frac{1}{q} w(t) \leqslant v(t) \leqslant w(t), \quad t \in[\underline{t}, \bar{t}]
$$

Consequently, since $t+\theta \in\left[t_{0}-\tau, \bar{t}\right]$ for all $t \in[\underline{t}, \bar{t}]$ and $\theta \in[-\tau, 0]$, we get from (13), (22), (25), and (27) that

$$
\begin{array}{r}
v(t+\theta) \leqslant w(t+\theta) \leqslant \mathrm{e}^{\alpha \tau} w(t) \leqslant q \mathrm{e}^{\alpha \tau} v(t) \leqslant \gamma v(t), \\
t \in[\underline{t}, \bar{t}], \theta \in[-\tau, 0] .
\end{array}
$$

By similar argument, we will be led to contradiction (21) once again, which verifies the validity of (24).

By mathematical induction, we conclude that (11) or equivalently, (8), is true. Then, it follows from condition (i) that

$$
\mathbb{E} h(t, x(t)) \leqslant C \mathbb{E} h_{0}\left(t_{0}, \xi\right) \mathrm{e}^{-\alpha\left(t-t_{0}\right)}, \quad t \geqslant t_{0},
$$

where $C=\max \left\{q c_{2} / c_{1} \prod_{k=1}^{\infty}\left(1+\beta_{k}\right), 1\right\}$. Thus, system (1) is $\left(h_{0}, h\right)$-exponentially stable with convergence rate $\alpha$. Furthermore, in view of the fact that $\beta>1$ is arbitrary, we must have $\alpha=\min \left\{\ln (\gamma / q) / \tau,\left(\ln q-M_{1} M_{2}\right) / \mu\right\}>0$. The proof is therefore complete.

Remark 6. It is noted that Theorem 5 allows for significant increases in $V$ between impulses as long as the decrease of $V$ at impulse times balance it properly. We can see that the impulses do contribute to the stability behavior of the system. Moreover, compared with the $p$ th-moment exponential stability theorem in [13] which assumed that

$$
\mathbb{E} V\left(t_{k}, I_{k}\left(t_{k}, \phi\right)\right) \leqslant \rho d_{k} \mathbb{E} V\left(t_{k}^{-}, \phi(0)\right),
$$

where $0<\rho<1, d_{k}>0, \prod_{k=1}^{\infty} d_{k}<\infty$, our result has a wider adaptive range.

Especially, the convergence rate $\alpha=0$ if $q=\gamma$ in Theorem 5, which implies that system $(1)$ is $\left(h_{0}, h\right)$-uniformly stable. Therefore, letting $q$ tend to $\gamma$ in Theorem 5 will immediately yield the following corollary.

Corollary 7. Assume that conditions (i)-(iii) of Theorem 5 hold, while condition (iv) is replaced by

$\left(i v^{*}\right) \ln \gamma>M_{1} M_{2}$, where $M_{1}=\sup _{t \geqslant 0} \int_{t}^{t+\mu} b(s) d s, M_{2}=$ $\sup _{s>0}\{c(s) / s\}$, and $\mu=\sup _{k \in \mathbb{N}}\left\{t_{k}-t_{k-1}\right\}$.

Then, system $(1)$ is $\left(h_{0}, h\right)$-uniformly stable for any time delay $\tau \in(0, \infty)$.

\section{Special Cases}

In this section, we will apply the general Razumikhin-type theorems established in previous section to deal with the stability of two special types of system (1).

Case 1 (ISFDSs-nDI). An important special case of system (1) is the following ISFDSs-nDI, in which the state variables on impulses are not related to the time delay

$$
\begin{gathered}
\mathrm{d} x(t)=f\left(t, x_{t}\right) \mathrm{d} t+\sigma\left(t, x_{t}\right) \mathrm{d} B(t), \quad t \geqslant t_{0}, \quad t \neq t_{k}, \\
x\left(t_{k}\right)=I_{k}\left(t_{k}, x\left(t_{k}^{-}\right)\right), \quad k \in \mathbb{N} .
\end{gathered}
$$


Theorem 8. Assume that all conditions of Theorem 5 hold with the following change:

(iii $\left.{ }^{*}\right) \mathbb{E} V\left(t_{k}, I_{k}\left(t_{k}, x\right)\right) \leqslant 1 / q\left(1+\beta_{k}\right) \mathbb{E} V\left(t_{k}^{-}, x\right)$, where $\beta_{k} \leqslant$ 0 and $\prod_{k=1}^{\infty} \beta_{k}<\infty$. Then, system (31) is $\left(h_{0}, h\right)$ exponentially stable for any time delay $\tau \in(0, \infty)$, and the convergence rate is not greater than $\min \{\ln (r /$ $\left.q) / \tau,\left(\ln q-M_{1} M_{2}\right) / \mu\right\}$.

Proof. The proof is similar to that of Theorem 5 only replacing (23) by

$$
\begin{aligned}
v\left(t_{m}\right) & \leqslant \frac{1}{q}\left(1+\beta_{m}\right) v\left(t_{m}^{-}\right) \leqslant \frac{1}{q}\left(1+\beta_{m}\right) w\left(t_{m}^{-}\right) \\
& =c_{2} \mathbb{E} h_{0}\left(t_{0}, \xi\right) H\left(t_{m}\right) \mathrm{e}^{-\alpha\left(t_{m}-t_{0}\right)}=\frac{1}{q} w\left(t_{m}\right)<w\left(t_{m}\right) .
\end{aligned}
$$

Remark 9. In a special case when $h(t, x)=h^{0}(t, x)=|x|^{p}$, $b(t) \equiv 1, c(s)=\gamma_{1}(s)$, Theorem 8 can be expressed as Theorem 3.1 in [25], which demonstrates the generality of our result.

Case 2 (IFDSs-DI). Deterministic systems may be regarded as a special class of stochastic systems. The following deterministic IFDSs-DI, which have been investigated in $[10,11]$, are exactly system $(1)$ with $\sigma(t, \phi) \equiv 0$ :

$$
\begin{gathered}
\dot{x}(t)=f\left(t, x_{t}\right), \quad t \geqslant t_{0}, \quad t \neq t_{k}, \\
x\left(t_{k}\right)=I_{k}\left(t_{k}, x_{t_{k}^{-}}\right), \quad k \in \mathbb{N} .
\end{gathered}
$$

Based on Theorem 5, one can easily get the following exponential stability result for system (33). For the notation of $v_{0}$ function family and the upper right-hand derivative appeared in the following theorem, we refer to [6].

Theorem 10. Assume that there exist functions $V \in v_{0}, h^{0}, h \in$ $\Gamma$ and constants $c_{1}>0, c_{2}>0, \gamma>q>1$ such that

(i) $c_{1} h(t, x) \leqslant V(t, x) \leqslant c_{2} h^{0}(t, x)$ for any $(t, x) \in\left[t_{0}-\right.$ $\tau, \infty) \times \mathbb{R}^{n}$

(ii) $D^{+} V(t, \phi(0)) \leqslant b(t) c(V(t, \phi(0)))$ for all $t \in\left[t_{k-1}, t_{k}\right)$, $k \in \mathbb{N}$ and those $\phi \in P C\left([-\tau, 0] ; \mathbb{R}^{n}\right)$ satisfying

$$
V(t+\theta, \phi(\theta)) \leqslant \gamma V(t, \phi(0)) \quad \text { on }-\tau \leqslant \theta \leqslant 0
$$

(iii) $V\left(t_{k}, I_{k}\left(t_{k}, \phi\right)\right) \leqslant(1 / \gamma)\left(1+\beta_{k}\right) \sup _{s \in[-\tau, 0]} V\left(t_{k}^{-}+s, \phi(s)\right)$, where $\beta_{k} \geqslant 0$ and $\sum_{k=1}^{\infty} \beta_{k}<\infty$;

(iv) $\ln q>M_{1} M_{2}$, where $M_{1}=\sup _{t \geqslant 0} \int_{t}^{t+\mu} b(s) d s, M_{2}=$ $\sup _{s>0}\{c(s) / s\}$, and $\mu=\sup _{k \in \mathbb{N}}\left\{t_{k}-t_{k-1}\right\}$.

Then, system (33) is $\left(h_{0}, h\right)$-exponentially stable for any time delay $\tau \in(0, \infty)$, and the convergence rate is not greater than $\min \left\{\ln (\gamma / q) / \tau,\left(\ln q-M_{1} M_{2}\right) / \mu\right\}$.
Remark 11. Obviously, Theorem 10 includes Theorem 3.1 in [10] as a special case. While in [11], the exponential stability theorem was obtained under the following condition:

$$
\begin{aligned}
& V\left(t_{k}, I_{k}\left(t_{k}, \phi\right)\right) \leqslant d_{k} V\left(t_{k}^{-}, \phi(0)\right) \\
& \text { where } 0<d_{k-1} \leqslant 1 \text { are constants, }
\end{aligned}
$$

which is simpler but stronger. Therefore, our results are more general and considerably less conservative.

\section{Impulsive Stabilization}

This section is devoted to designing a delayed impulsive controller which mean square exponentially stabilize a linear stochastic delay system.

Suppose that we are given an $n$-dimensional linear stochastic system with time-varying delay

$$
\begin{aligned}
\mathrm{d} x(t)= & {\left[A_{0} x(t)+A_{1} x(t-\tau(t))\right] \mathrm{d} t } \\
& +\left[D_{0} x(t)+D_{1} x(t-\tau(t))\right] \mathrm{d} B(t), \quad t \geqslant t_{0},
\end{aligned}
$$

where $x(t) \in \mathbb{R}^{n}$ is the state vector; time delay of the system $\tau(t)$ is a Borel-measurable function on $t \geqslant 0$ with $0 \leqslant \tau(t) \leqslant$ $\tau$, where $\tau$ is a positive constant; and $B(t)$ is a standard onedimensional Brownian motion. We are required to stabilize system (36) by delayed impulses of the form

$$
x\left(t_{k}\right)=C_{0} x\left(t_{k}^{-}\right)+C_{1} x\left(t_{k}^{-}-\tau\left(t_{k}^{-}\right)\right), \quad k \in \mathbb{N} .
$$

In other words, we need to find appropriate impulsive gain matrices $C_{0}, C_{1}$ and impulse time sequence $\left\{t_{k}\right\}_{k \in \mathbb{N}}$ such that the corresponding linear impulsive stochastic delay system

$$
\begin{array}{r}
\mathrm{d} x(t)=\left[A_{0} x(t)+A_{1} x(t-\tau(t))\right] \mathrm{d} t \\
+\left[D_{0} x(t)+D_{1} x(t-\tau(t))\right] \mathrm{d} B(t), \\
t \geqslant t_{0}, \quad t \neq t_{k}, \\
x\left(t_{k}\right)=C_{0} x\left(t_{k}^{-}\right)+C_{1} x\left(t_{k}^{-}-\tau\left(t_{k}^{-}\right)\right), \quad k \in \mathbb{N} .
\end{array}
$$

Will be mean square exponentially stable.

Theorem 12. System (36) can be mean square exponentially stabilized by impulses in the form of (37) if there exist matrices 
$C_{i}, i=0,1, P>0$ and positive scalars $\varepsilon, \varepsilon_{j}, j=1,2,3,4$ such that the following matrix inequalities hold

$$
\begin{aligned}
& {\left[\begin{array}{rr}
P A_{0}+A_{0}^{\mathrm{T}} P+D_{0}^{\mathrm{T}} P D_{0}-\varepsilon_{1} P & P A_{1}+D_{0}^{\mathrm{T}} P D_{1} \\
& D_{1}^{\mathrm{T}} P D_{1}-\varepsilon_{2} P
\end{array}\right] \leqslant 0} \\
& {\left[\begin{array}{ccc}
-\varepsilon_{3} P & 0 & C_{0}^{\mathrm{T}} \\
* & -\varepsilon_{4} P & C_{1}^{\mathrm{T}} \\
* & * & -P^{-1}
\end{array}\right] \leqslant 0,} \\
& \varepsilon_{3}+\varepsilon_{4}<\varepsilon<1, \\
& \mu=\sup _{k \in \mathbb{N}}\left\{t_{k}-t_{k-1}\right\}<\frac{\left(\varepsilon_{3}+\varepsilon_{4}\right) \ln \left(\varepsilon /\left(\varepsilon_{3}+\varepsilon_{4}\right)\right)}{\varepsilon_{1}\left(\varepsilon_{3}+\varepsilon_{4}\right)+\varepsilon_{2}},
\end{aligned}
$$

where entries denoted by * can be readily inferred from the symmetry of a matrix.

Proof. The conclusion follows from Theorem 5 by considering $V(t, x)=x^{\mathrm{T}} P x$ and $h^{0}(t, x)=h(t, x)=|x|^{2}$.

Although (39)-(40) are linear with respect to $\left(C_{i}, \varepsilon_{j}\right), i=$ $0,1, j=1,2,3,4$ for fixed $P$, it is not linear with the combined variables $\left(C_{i}, \varepsilon_{j}, P\right)$, and, therefore, it is not LMIs. This makes the computation difficult but flexible. Two different impulsive controller designing steps are given as follows.

\section{Method I}

Step 1. Choose matrix $P>0$.

Step 2. Get $\varepsilon_{i}, i=1,2,3,4$ and $C_{0}, C_{1}$ by solving LMI (39), (40), and

$$
\begin{gathered}
\varepsilon_{i}>0, \quad i=1,2,3,4, \quad \varepsilon_{3}+\varepsilon_{4}<1, \\
C_{j}>(<) 0, \quad j=0,1 .
\end{gathered}
$$

Step 3. Choose $\left\{t_{k}\right\}_{k \in \mathbb{N}}$ such that $t_{k}-t_{k-1}<\left(\left(\varepsilon_{3}+\varepsilon_{4}\right) \ln \left(\varepsilon /\left(\varepsilon_{3}+\right.\right.\right.$ $\left.\left.\left.\varepsilon_{4}\right)\right)\right) /\left(\varepsilon_{1}\left(\varepsilon_{3}+\varepsilon_{4}\right)+\varepsilon_{2}\right), k \in \mathbb{N}$, where $\varepsilon$ is an arbitrary number satisfying $\varepsilon_{3}+\varepsilon_{4}<\varepsilon<1$.

\section{Method II}

Step 1. Give appropriate positive scalars $\varepsilon_{1}$ and $\varepsilon_{2}$ such that LMI (39) is feasible and get matrix $P>0$.

Step 2. Get $\varepsilon_{3}, \varepsilon_{4}$ and $C_{0}, C_{1}$ by solving LMI (40) and

$$
\begin{gathered}
\varepsilon_{i}>0, \quad i=3,4, \quad \varepsilon_{3}+\varepsilon_{4}<1, \\
C_{j}>(<) 0, \quad j=0,1 .
\end{gathered}
$$

Step 3. Choose $\left\{t_{k}\right\}_{k \in \mathbb{N}}$ such that $t_{k}-t_{k-1}<\left(\left(\varepsilon_{3}+\varepsilon_{4}\right) \ln \left(\varepsilon /\left(\varepsilon_{3}+\right.\right.\right.$ $\left.\left.\left.\varepsilon_{4}\right)\right)\right) /\left(\varepsilon_{1}\left(\varepsilon_{3}+\varepsilon_{4}\right)+\varepsilon_{2}\right), k \in \mathbb{N}$, where $\varepsilon$ is an arbitrary number satisfying $\varepsilon_{3}+\varepsilon_{4}<\varepsilon<1$.
Remark 13. Obviously, the stabilizing impulses (37) are not unique. For example, $C_{0}=C_{1}=0$ and $\left\{t_{k}\right\}_{k \in \mathbb{N}}$ with the first impulse interval being finite will be a simple one. In the above two methods, we impose conditions $C_{i}>0$ or $C_{i}<0$ to get impulsive controllers with $C_{i} \neq 0, i=0,1$.

\section{Illustrative Examples}

In this section, examples are given to verify the effectiveness and advantages of our results. For simplicity, we consider the case of constant delay in the following examples. It should be pointed out that our results can be applied to systems with time-varying delay.

Example 14. Let us consider a two-dimensional impulsive stochastic delay system

$$
\begin{aligned}
& \mathrm{d} x_{1}(t)= x_{1}(t) \mathrm{d} t+x_{1}(t) \mathrm{d} B(t), \quad t \geqslant t_{0}, \quad t \neq t_{k}, \\
& \mathrm{~d} x_{2}(t)= {\left[-x_{1}^{2}(t-\tau) x_{2}(t)-\frac{1}{2} a x_{2}(t)\right] \mathrm{d} t } \\
&-\sqrt{a} x_{2}(t-\tau) \mathrm{d} B(t), \quad t \geqslant t_{0}, \quad t \neq t_{k}, \\
& x_{1}\left(t_{k}\right)=x_{1}\left(t_{k}^{-}\right), \quad k \in \mathbb{N}, \\
& x_{2}\left(t_{k}\right)= \frac{1}{2 \sqrt{\gamma}}\left(1+k^{-2}\right)\left[x_{2}\left(t_{k}^{-}\right)+x_{2}\left(t_{k}^{-}-\tau\right)\right], \\
& k \in \mathbb{N},
\end{aligned}
$$

where constants $a>0, \tau>0$, and $\gamma>1$. If

$$
\mu=\sup _{k \in \mathbb{N}}\left\{t_{k}-t_{k-1}\right\}<\frac{\ln q}{a(\gamma-1)},
$$

for any $q$ satisfying $1<q<\gamma$, then system (45) is mean square exponentially stable with respect to $x_{2}$. If

$$
\mu<\frac{\ln \gamma}{a(\gamma-1)}
$$

then system (45) is mean square uniformly stable with respect to $x_{2}$.

We first note that the $\left(h_{0}, h\right)$-stability properties reduce to the mean square stability properties with respect to $x_{2}$ if $h^{0}(t, x)=x_{1}^{2}+x_{2}^{2}, h(t, x)=x_{2}^{2}$. Choose $V(x)=x_{2}^{2}$, then

$$
\begin{aligned}
& \mathbb{E} V\left(x\left(t_{k}\right)\right) \\
& =\frac{1}{4 \gamma}\left(1+k^{-2}\right)^{2} \mathbb{E}\left[x_{2}\left(t_{k}^{-}\right)+x_{2}\left(t_{k}^{-}-\tau\right)\right]^{2} \\
& \quad \leqslant \frac{1}{\gamma}\left(1+k^{-4}+2 k^{-2}\right) \sup _{s \in[-\tau, 0]} \mathbb{E} V\left(x\left(t_{k}^{-}+s\right)\right), \\
& \mathbb{E} \mathscr{L} V\left(x_{t}\right) \\
& \quad=\mathbb{E}\left[-2 x_{1}^{2}(t-\tau) x_{2}^{2}(t)-a x_{2}^{2}(t)+a x_{2}^{2}(t-\tau)\right] \\
& \leqslant a(\gamma-1) \mathbb{E} x_{2}^{2}(t)=a(\gamma-1) \mathbb{E} V(x(t)),
\end{aligned}
$$

whenever $\mathbb{E} V(x(t+s)) \leqslant \gamma(\mathbb{E} V(x(t))),-\tau \leqslant s \leqslant 0$. 


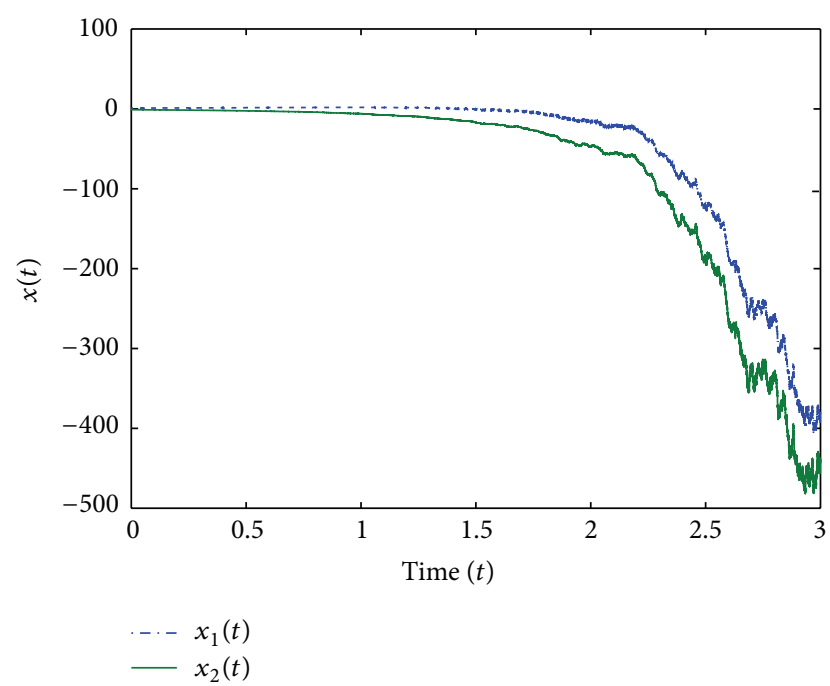

FIGURE 1: The solution to system (51) without impulses (single sample).

Taking $c_{1}=c_{2}=1, b(t) \equiv 1, c(s)=a(\gamma-1) s$, and $\beta_{k}=k^{-4}+$ $2 k^{-2}$, it is easy to check that all the conditions of Theorem 5 are satisfied under condition (46), which means that system (45) is mean square exponentially stable with respect to $x_{2}$. As well, under condition (47), one can derive the mean square uniform stability with respect to $x_{2}$ according to Corollary 7 .

It is noted that

$$
x_{1}(t)=x_{10} \exp \left[\frac{1}{2\left(t-t_{0}\right)}+B(t)-B\left(t_{0}\right)\right] \text {, }
$$

where $x_{10}$ is the initial data of $x_{1}(t)$. Thus,

$$
\mathbb{E}\left|x_{1}(t)\right|^{2}=\left|x_{10}\right|^{2} \mathrm{e}^{t-t_{0}},
$$

which demonstrates that the state $x_{1}$ is mean square exponentially unstable. Hence, the existing stability theorems for the trivial solution fail to work. This shows that the notion of stability in terms of two measures is more general, and our results have a wider adaptive range.

Example 15. Consider the following stochastic delay system

$$
\begin{aligned}
\mathrm{d} x(t)= & {\left[A_{0} x(t)+A_{1} x(t-\tau)\right] \mathrm{d} t } \\
& +\left[D_{0} x(t)+D_{1} x(t-\tau)\right] \mathrm{d} B(t), \quad t \geqslant t_{0},
\end{aligned}
$$

where $A_{0}=\left[\begin{array}{cc}0.5 & 0.3 \\ 0 & 0.4\end{array}\right], A_{1}=\left[\begin{array}{cc}1.3 & 0.3 \\ 0.23 & 1.5\end{array}\right], D_{0}=\left[\begin{array}{cc}0.2 & 0.1 \\ 0.3 & 0.1\end{array}\right]$, and $D_{1}=$ $0.1 I$.

It is noted that system (51) is not stable, and that its simulations with $\tau=0.002$, initial data $\xi(\theta)=[1-1]^{\mathrm{T}}$, and $\theta \in[-0.002,0]$ are shown in Figures 1 and 2 . In the following, we will design a delayed impulsive control law according to Theorem 12 to exponentially stabilize system (51). Adopting Method I, we choose $P=3.25 I, \varepsilon=0.9999$, then

$$
\begin{aligned}
& \varepsilon_{1}=3.6290, \quad \varepsilon_{2}=3.0558, \quad \varepsilon_{3}=0.7334, \\
& \varepsilon_{4}=0.1929, \quad C_{0}=0.8089 I, \quad C_{1}=0.1110 I
\end{aligned}
$$

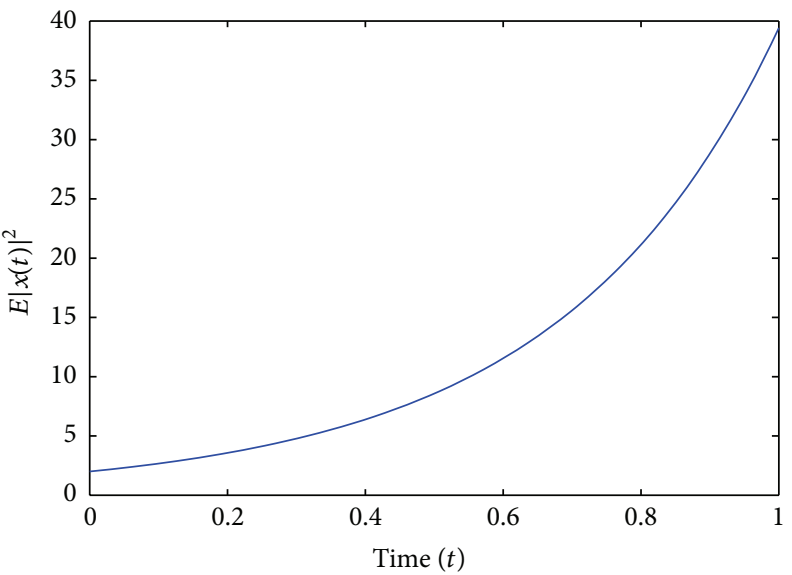

FIGURE 2: The mean square of the solution to system (51) without impulses (2000 samples).

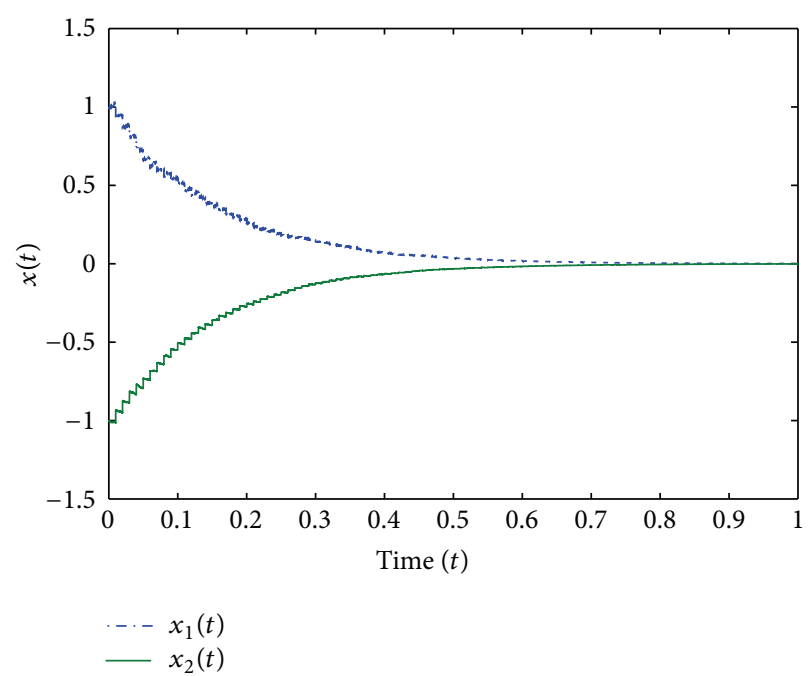

FIGURE 3: The solution to system (51) with impulses (single sample).

is a group of feasible solution to linear matrix inequalities (39)-(41), and the maximum impulse interval can be chosen as

$$
\mu=0.01<\frac{\left(\varepsilon_{3}+\varepsilon_{4}\right) \ln \left(\varepsilon /\left(\varepsilon_{3}+\varepsilon_{4}\right)\right)}{\varepsilon_{1}\left(\varepsilon_{3}+\varepsilon_{4}\right)+\varepsilon_{2}}=0.011 .
$$

The simulations of system (51) under impulsive control are displayed in Figures 3 and 4 , where $\tau=0.002$, initial data $\xi(\theta)=[1-1]^{\mathrm{T}}, \theta \in[-0.002,0]$, and impulse interval $t_{k}-$ $t_{k-1}=0.01, k \in \mathbb{N}$. It is clearly demonstrated that the impulses we designed successfully stabilize an unstable stochastic delay system.

\section{Conclusion}

This paper has investigated the exponential stability in terms of two measures for ISFDSs-DI based on Razumikhin-type arguments. The results obtained improve and complement some recent works. Moreover, the stability criteria obtained 


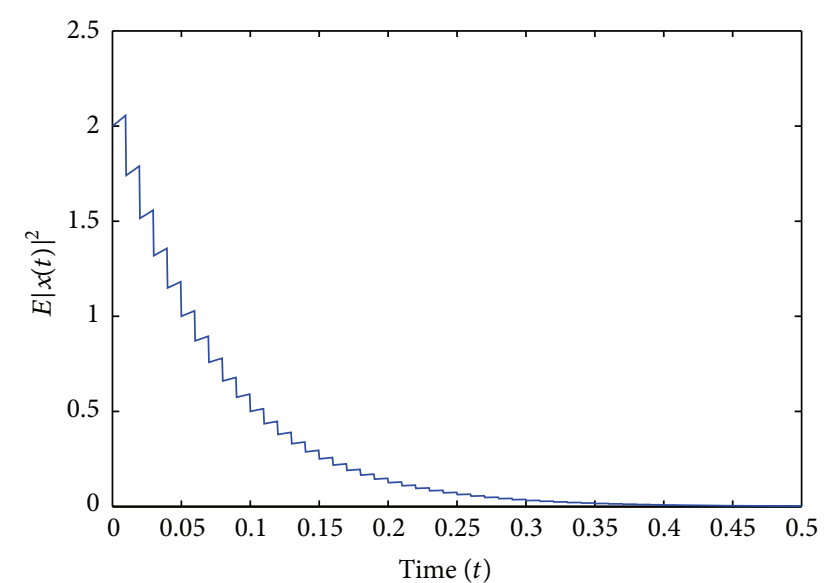

FIGURE 4: The mean square of the solution to system (51) with impulses (2000 samples).

are applied to stabilize linear stochastic delay systems, and delayed impulsive controllers that exponentially stabilize the systems are proposed.

\section{Acknowledgments}

This work was supported by the National Natural Science Foundation of China under Grants 660874114, 61273126, and 11226247 and the Anhui Provincial Nature Science Foundation 1308085QA15.

\section{References}

[1] Z. Luo and J. Shen, "Impulsive stabilization of functional differential equations with infinite delays," Applied Mathematics Letters, vol. 16, no. 5, pp. 695-701, 2003.

[2] X. Liu, "Stability of impulsive control systems with time delay," Mathematical and Computer Modelling, vol. 39, no. 4-5, pp. 511519, 2004.

[3] X. Liu and Q. Wang, "The method of Lyapunov functionals and exponential stability of impulsive systems with time delay," Nonlinear Analysis: Theory, Methods \& Applications, vol. 66, no. 7, pp. 1465-1484, 2007.

[4] X. Liu and Q. Wang, "On stability in terms of two measures for impulsive systems of functional differential equations," Journal of Mathematical Analysis and Applications, vol. 326, no. 1, pp. 252-265, 2007.

[5] Q. Wang and X. Liu, "Impulsive stabilization of delay differential systems via the Lyapunov-Razumikhin method," Applied Mathematics Letters, vol. 20, no. 8, pp. 839-845, 2007.

[6] X. Li, "New results on global exponential stabilization of impulsive functional differential equations with infinite delays or finite delays," Nonlinear Analysis: Real World Applications, vol. 11, no. 5, pp. 4194-4201, 2010.

[7] X. Fu and X. Li, "Razumikhin-type theorems on exponential stability of impulsive infinite delay differential systems," Journal of Computational and Applied Mathematics, vol. 224, no. 1, pp. 1-10, 2009.

[8] W.-H. Chen and W. X. Zheng, "Robust stability and $H_{\infty}$-control of uncertain impulsive systems with time-delay," Automatica, vol. 45, no. 1, pp. 109-117, 2009.
[9] Y. Zhang and J. Sun, "Stability of impulsive functional differential equations," Nonlinear Analysis: Theory, Methods \& Applications, vol. 68, no. 12, pp. 3665-3678, 2008.

[10] D. Lin, X. Li, and D. O'Regan, "Stability analysis of generalized impulsive functional differential equations," Mathematical and Computer Modelling, vol. 55, no. 5-6, pp. 1682-1690, 2012.

[11] Q. Wu, J. Zhou, and L. Xiang, "Global exponential stability of impulsive differential equations with any time delays," Applied Mathematics Letters, vol. 23, no. 2, pp. 143-147, 2010.

[12] A. Khadra, X. Z. Liu, and X. Shen, "Analyzing the robustness of impulsive synchronization coupled by linear delayed impulses," Institute of Electrical and Electronics Engineers, vol. 54, no. 4, pp. 923-928, 2009.

[13] J. Liu, X. Liu, and W.-C. Xie, "Impulsive stabilization of stochastic functional differential equations," Applied Mathematics Letters, vol. 24, no. 3, pp. 264-269, 2011.

[14] P. Cheng, F. Deng, and Y. Peng, "Robust exponential stability and delayed-state-feedback stabilization of uncertain impulsive stochastic systems with time-varying delay," Communications in Nonlinear Science and Numerical Simulation, vol. 17, no. 12, pp. 4740-4752, 2012.

[15] T. Yang and L. O. Chua, "Impulsive stabilization for control and synchronization of chaotic systems: theory and application to secure communication," IEEE Transactions on Circuits and Systems. I, vol. 44, no. 10, pp. 976-988, 1997.

[16] X. Liu, "Impulsive stabilization and control of chaotic system," Nonlinear Analysis: Theory, Methods \& Applications, vol. 47, pp. 1081-1092, 2001.

[17] C. Li, X. Liao, X. Yang, and T. Huang, "Impulsive stabilization and synchronization of a class of chaotic delay systems," Chaos, vol. 15, no. 4, Article ID 043103, 9 pages, 2005.

[18] X. Liu and K. Rohlf, "Impulsive control of a Lotka-Volterra system," IMA Journal of Mathematical Control and Information, vol. 15, no. 3, pp. 269-284, 1998.

[19] X. Liu and A. R. Willms, "Impulsive controllability of linear dynamical systems with applications to maneuvers of spacecraft," Mathematical Problems in Engineering, pp. 277-299, 1996.

[20] T. E. Carter, "Optimal impulsive space trajectories based on linear equations," Journal of Optimization Theory and Applications, vol. 70, no. 2, pp. 277-297, 1991.

[21] N. Yu-Jun, X. Wei, and L. Hong-Wu, "Asymptotical p-moment stability of stochastic impulsive differential equations and its application in impulsive control," Communications in Theoretical Physics, vol. 53, no. 1, pp. 110-114, 2010.

[22] C. Li, L. Chen, and K. Aihara, "Impulsive control of stochastic systems with applications in chaos control, chaos synchronization, and neural networks," Chaos, vol. 18, no. 2, Article ID 023132, 11 pages, 2008.

[23] L. Huang and X. Mao, "Robust delayed-state-feedback stabilization of uncertain stochastic systems," Automatica, vol. 45, no. 5, pp. 1332-1339, 2009.

[24] M. S. Alwan, X. Liu, and W.-C. Xie, "Existence, continuation, and uniqueness problems of stochastic impulsive systems with time delay," Journal of the Franklin Institute, vol. 347, no. 7, pp. 1317-1333, 2010.

[25] S. Peng and Y. Zhang, "Razumikhin-type theorems on $p$ th moment exponential stability of impulsive stochastic delay differential equations," Institute of Electrical and Electronics Engineers, vol. 55, no. 8, pp. 1917-1922, 2010.

[26] V. Lakshmikantham and X. Z. Liu, Stability Analysis in Terms of Two Measures, World Scientific, River Edge, NJ, USA, 1993. 


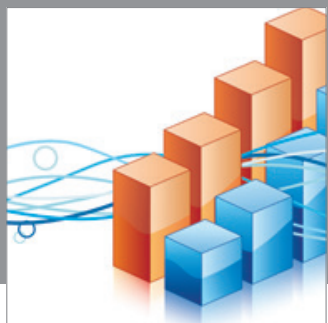

Advances in

Operations Research

mansans

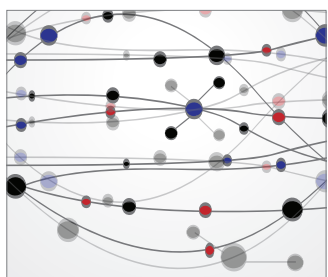

The Scientific World Journal
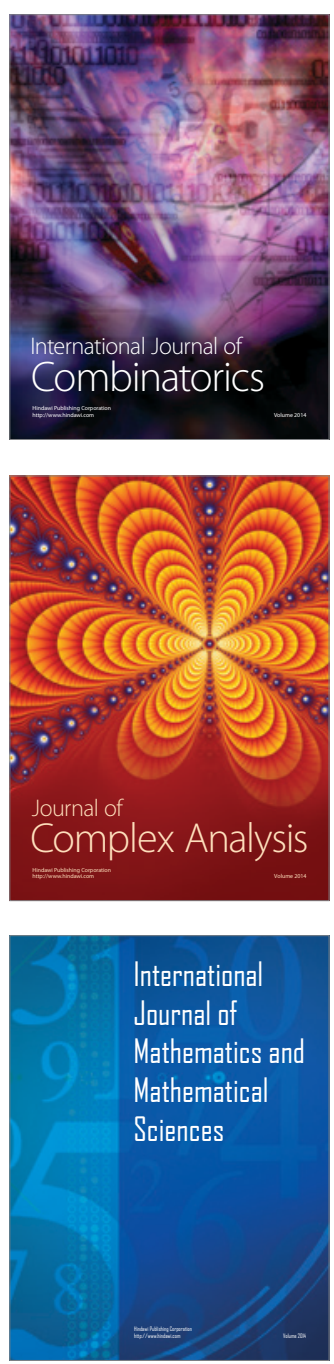
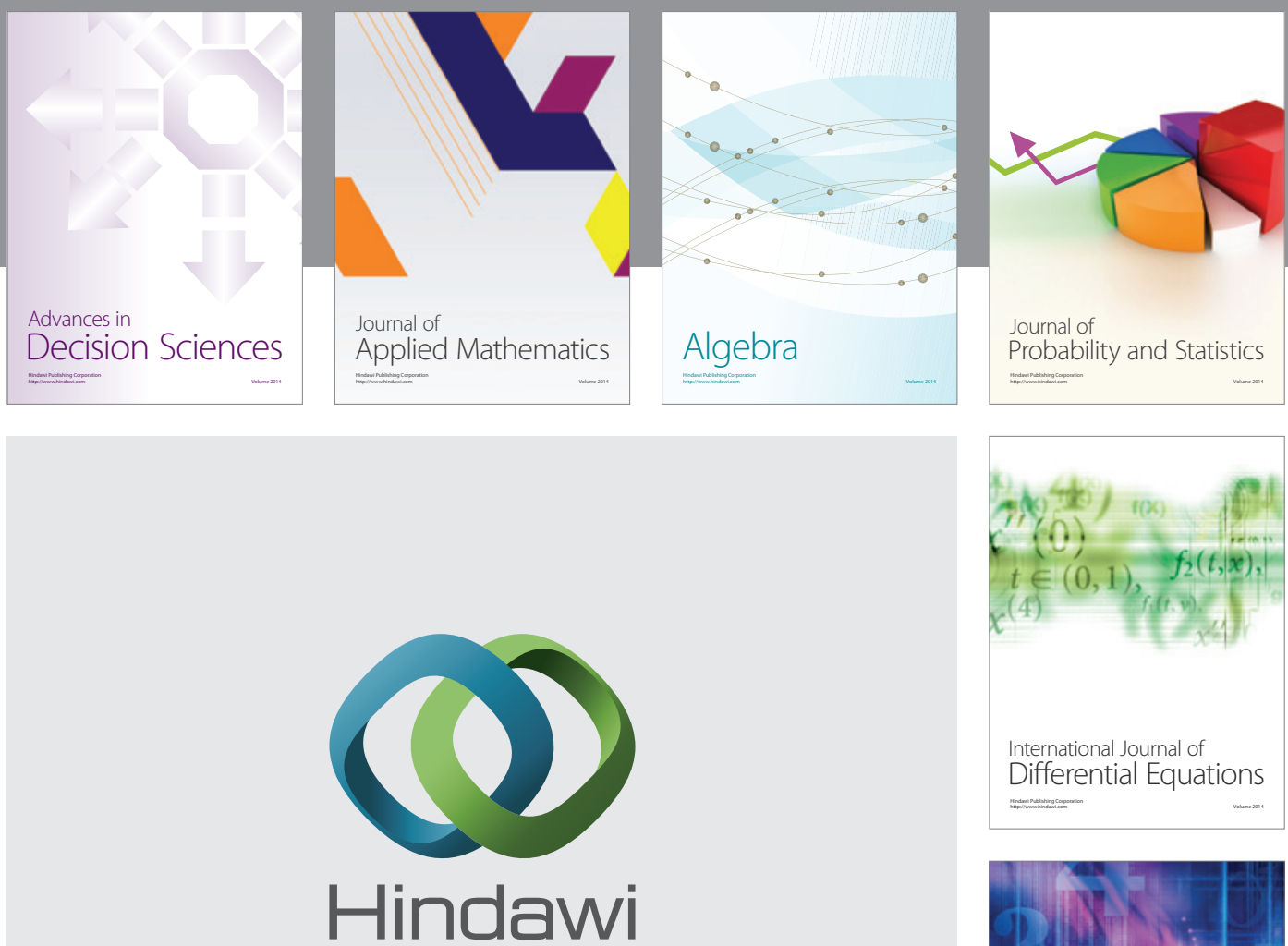

Submit your manuscripts at http://www.hindawi.com
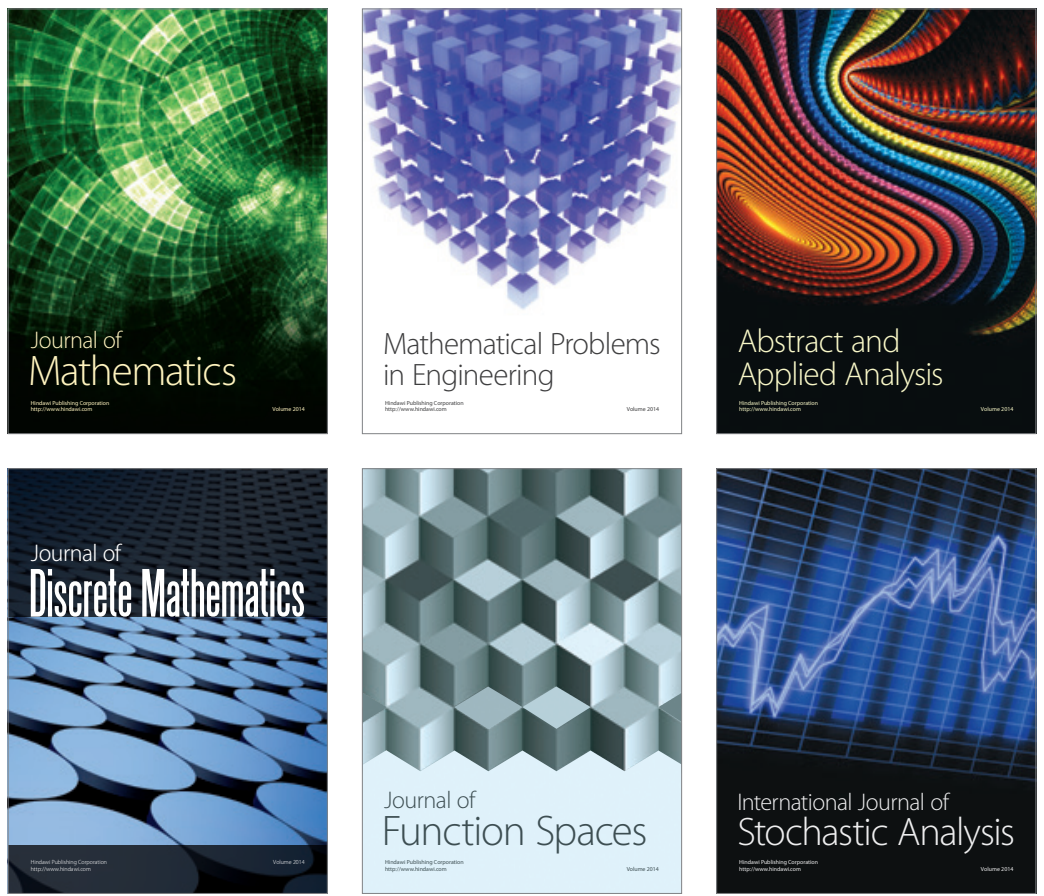

Journal of

Function Spaces

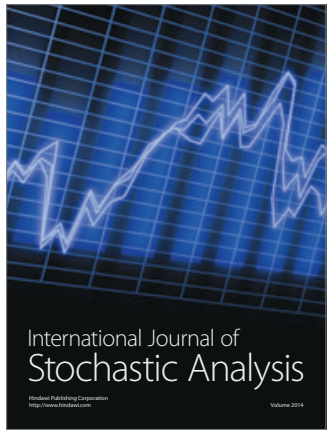

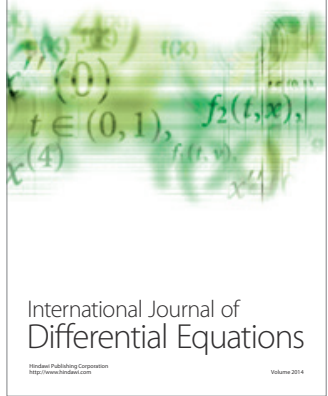
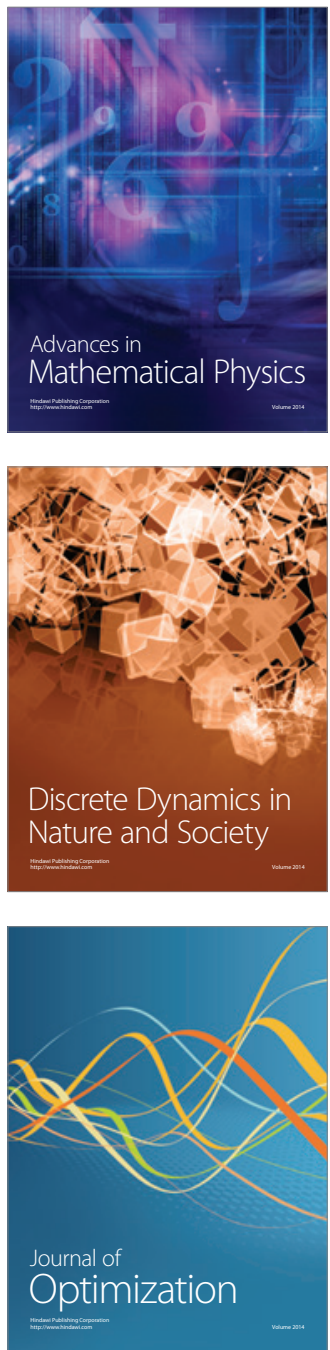